\title{
CHEMICAL EVOLUTION OF THE GALACTIC DISK AND THE RADIAL METALLICITY GRADIENT
}

\author{
M. MAYOR \\ Observatoire de Genève, Switzerland
}

Abstract. An analysis of the kinematical and photometric properties of about $600 \mathrm{dF}$ stars and $600 \mathrm{gG}-\mathrm{gK}$ stars permits the estimation of the radial chemical gradient in the Galaxy. The mean value in the solar neighbourhood obtained for all of these stars is:

$$
\frac{\partial[\mathrm{Fe} / \mathrm{H}]}{\partial \widetilde{\omega}}=-0.05 \pm 0.01 \mathrm{kpc}^{-1}
$$

The values of $[\mathrm{Fe} / \mathrm{H}]$ used for this estimation are deduced for the $\mathrm{dF}$ stars using uvby $\beta$ photometric measurements and for the $\mathrm{gG}-\mathrm{gK}$ stars from a list published by Hansen and Kjaergaard. An estimate of the chemical gradient using $U B V$ photometry of dG stars in the solar neighbourhood gives a similar value. For all the samples studied $(\mathrm{dF}, \mathrm{dG}$ or giants) the order of magnitude for the gradient is the same. However, for the youngest stars in these samples the metallicity gradient could be larger:

$$
\frac{\partial[\mathrm{Fe} / \mathrm{H}]}{\partial \widetilde{\omega}}=-0.10 \pm 0.02 \mathrm{kpc}^{-1}
$$

Such a value may be affected by dynamical perturbations of the galactic disk.

The values published by Hansen and Kjaergaard for the sodium concentration in giant star atmospheres also indicate a radial galactic gradient of the same order.

If only the $\mathrm{dF}$ stars which are sufficiently evolved to allow an age estimate are considered, then a very distinct correlation is found between age and metallicity:

$$
\frac{\partial \bar{Z} / \bar{Z}_{\odot}}{\partial t}=0.6 \pm 0.3 / 10^{10} \mathrm{yr}
$$

An important fraction of the heavy elements actually present in the solar neighbourhood seems to have synthetized during the life of the galactic disk.

The two derivatives $\frac{\partial \bar{Z}}{\partial t}$ and $\frac{\partial Z}{\partial \widetilde{\omega}}$ are not independent, but are connected by the chemical evolution of the galactic disk. Some elementary deductions show the coherency of these two estimates.

The intrinsic dispersion of metallicities, at a given age and birthplace, is somewhat lower than the admitted values.

$$
\left.\sigma_{[\mathrm{Fe} / \mathrm{H}]}\right|_{\widetilde{\omega}, t}=0.10\left(\begin{array}{c}
+0.05 \\
-0.10
\end{array}\right)
$$


It has not been possible to find any significant variation with age of this quantity from the present observational material. The simultaneous variation of $\sigma_{w}^{2}$ and $[\mathrm{Fe} / \mathrm{H}]$ as function of age is evidence for a $z$ stratification in the mean abundance of the heavy elements. The ratio between the mean metallicity in the plane and at $z=500 \mathrm{pc}$ is estimated to be about a factor of two.

Finally it is shown that the interpretation of the kinematical diagrams for different groups of given metallicity is ambiguous. A relation as $e$ vs $[\mathrm{Fe} / \mathrm{H}]$ depends not only on the chemical and kinematical history of the Galaxy but is also strongly dependent on the observational errors of $[\mathrm{Fe} / \mathrm{H}]$ and on criteria used to define the sample.

A paper containing the above results has been submitted for publication in Astronomy and Astrophysics.

\section{DISCUSSION}

Gerbaldi: Did you have some Fm stars in your statistic? In view that those stars are at the cool end of the Am phenomena and that the metallicity is produced by diffusion, i.e. in a quite short time regarding the evolution time, the presence of these stars could introduce some disturbance in your results.

Mayor: All stars with. noted peculiarities in the B5 catalogue have been excluded but evidently it is possible that a small number of unrecognized Fm stars be present.

Bell: Do you plan to make use of Maeder's evolutionary tracks, which give a better fit to cluster main sequences?

Mayor: At the time of the beginning of this investigation the evolutionary tracks of Maeder were not available but $I$ intend to use them. 